\title{
Contribution of dynamic sentinel lymphoscintigraphy images to the diagnosis of patients with malignant skin neoplasms in the upper and lower extremities
}

Hiroyuki Miura ${ }^{1 *}$, Shuichi Ono ${ }^{1}$, Koichi Shibutani ${ }^{1}$, Hiroko Seino ${ }^{1}$, Fumiyasu Tsushima ${ }^{1}$, Shinya Kakehata ${ }^{1}$, Katsumi Hirose ', Hiromasa Fujita', Akihisa Kakuta', Masahiko Aoki', Yoshiomi Hatayma', Hideo Kawaguchi', Mariko Sato ${ }^{1}$, Yoshihiro Takai ${ }^{1}$, Takahide Kaneko ${ }^{2}$ and Daisuke Sawamura ${ }^{2}$

\begin{abstract}
The aim of the present study was to confirm the contribution of dynamic images in sentinel lymphoscintigraphy in malignant skin neoplasms: precisely, to investigate if dynamic images were necessary and to observe if dynamic images could reduce the areas needed for biopsy and dissection. Twenty-five patients with malignant skin neoplasms of the lower $(n=21)$ and upper $(n=4)$ extremities were retrospectively investigated. Images were evaluated by two independent reviewers, an expert in diagnostic radiology and nuclear medicine and a diagnostic radiologist in training. Visualized hot spots were assessed to be sentinel nodes using only static planar images. Next, both static planar and dynamic images were assessed. Reviewers scored diagnostic confidence values of determined sentinel nodes as follows: 0, cannot be decided; 1, possible; 2, probable; and 3, definitive. Patterns of lymphatic drainage were categorized into six different pathways: (1) inguinal type, (2) popliteal type, (3) inguinal and popliteal type, (4) axillary type, (5) cubital type, and (6) axillary and cubital type. In cases in the lower extremities, with dynamic images, the expert reviewer changed assessment in three cases and the trainee reviewer changed it in one case. There were no cases in which a decision was changed to be the same between both reviewers. Although the average diagnostic confidence value of assessment is usually higher with dynamic images, significant differences were not present. In cases of the upper extremities, both reviewers changed their assessment in one patient. By mutual agreement, cases in which assessment was changed with dynamic images were the inguinal and popliteal type, and the axillary and cubital type. The expert reviewer noticed lymphatic channels only visualized on dynamic images and changed assessment. Determination of whether or not a lymph node is a sentinel node depends on visualization of the lymphatic network. In the present circumstances, all biopsies of hot spots determined to be lymph nodes should not be excluded. However, excessive biopsies should be avoided as much as possible. It is necessary to use dynamic images alongside skillful observation.
\end{abstract}

Keywords: Sentinel lymphoscintigraphy; Dynamic image; Malignant skin neoplasm; Upper and lower extremities

\footnotetext{
* Correspondence: miuradio@cc.hirosaki-u.ac.jp

${ }^{1}$ Department of Radiology, Hirosaki University School of Medicine, 5

Zaifu-cho, Hirosaki, Aomori 036-8562, Japan

Full list of author information is available at the end of the article
} 


\section{Introduction}

Radioguided sentinel lymph node detection in malignant skin neoplasms is currently both common and indispensable (Gennari et al. 2000; Jacobs et al. 2001; Belhocine et al. 2002a; Alazraki et al. 2002; Rossi et al. 2006; Mariani et al. 2002; Mariani et al. 2004; Uren et al. 1996). In cases of malignant cutaneous neoplasms in the arms and legs, sentinel lymphoscintigraphy can occasionally reveal lymph nodes in the poples, cubitus, and several other unexpected areas (Uren et al. 2003; Vidal-Sicart et al. 2003; Vidal-Sicart et al. 2004; Tiffet et al. 2004; Matter et al. 2007) which are called interval nodes, in-transit lymph nodes, ectopic lymph nodes, and so on (Matter et al. 2007). The risk of metastasis to an interval node is similar to that of other sentinel lymph nodes, and the interval node may be the only metastatic site (Matter et al. 2007). Therefore, searching such areas should not be excluded in sentinel lymph node detection. Conversely, if all downstream lymph nodes in the groin and axilla are second echelon lymph nodes, interval node biopsy alone may be sufficient. This abbreviated lymph node dissection should reduce the invasiveness of the investigation. However, in some cases, there are lymphatic channels that are visualized only in early phase dynamic images immediately after tracer injection (Taylor et al. 1996; Maza et al. 2003; Toubert et al. 2008; Miura et al. 2010). If dynamic images are not observed, assessment of the sentinel lymph node might be different in these cases (Matter et al. 2007; Toubert et al. 2008; Miura et al. 2010). Therefore, it is important to determine if the hot spot is a sentinel lymph node, as this can be closely related to the patient's prognosis (Gennari et al. 2000; Taylor et al. 1996; Maza et al. 2003). The aim of the present study was to confirm the contribution of dynamic images in sentinel lymphoscintigraphy: precisely, to investigate if dynamic images were necessary and to observe if dynamic images could reduce the areas needed for biopsy and lymph node dissection.

\section{Materials and methods Patients}

Twenty-five patients with malignant skin neoplasms of the lower and upper extremities (10 male, 15 female; age range 51-84 years, mean age 68.6 years) were retrospectively investigated. Of the 25 patients, 21 had malignant cutaneous neoplasms in the upper extremities, and four had malignant cutaneous neoplasms in the lower extremities (Table 1). There were no cases with a prior history of serious external injury which could have brought about a change in lymphatic drainage. This sentinel lymph node detection by nuclear medicine was performed based on the guidelines of the Japanese Society of Nuclear Medicine and approved by the ethics committee of the in-hospital, and these patients were provided informed consent in writing.

\section{Imaging procedure}

Approximately $150 \mathrm{MBq} / 0.5 \mathrm{~mL}$ of ${ }^{99 \mathrm{~m}} \mathrm{Tc}$-phytate (FUJIFILM RI Pharma Co., Ltd., former Daiichi Radioisotope Laboratory Co., Ltd., Japan) was injected intracutaneously around the primary tumor or biopsy scar (Uren et al. 2003; Glass et al. 1998; Belhocine et al. 2002). Typically, four injections were required, although the number of injections depended on the size or location of the primary tumor or scar (Uren et al. 2003).

First, a dynamic scan of 10-20 min was aquired immediately after radiotracer injection (Pijpers et al. 1995; Murray et al. 2000). Next, anterior and posterior views of static scans were imaged approximately $20 \mathrm{~min}$ after tracer injection. At first, a dynamic scan of 10-20 min was performed immediately after radiotracer injection using a low-energy high-resolution (LEHR) collimator (MULTISPECT 2 gamma camera; Siemens, Germany) at one frame per $30 \mathrm{~s}$ for 30 frames with a $128 * 128$ matrix. After the dynamic scan, about 20 min after tracer injection, static scans (anterior and posterior views, and oblique view if necessary) were performed for 3-5 min with a $512 * 512$ matrix, using the LEHR collimator. Later, the gamma camera was renewed and a new imaging protocol was carried out as follows: a dynamic scan for 10-20 min was performed immediately after radiotracer injection using a LEHR collimator (Infinia Hawkeye gamma camera; GE Healthcare, USA) at one frame per $30 \mathrm{~s}$, for 40 frames, with a $128 * 128$ matrix. After the dynamic scan, about 20 min after tracer injection, static scans (anterior and posterior views, and oblique view if necessary) were performed for 3 min with a $512 * 512$ matrix, using the LEHR collimator.

\section{Imaging observations}

Obtained images were observed and evaluated by two independent reviewers. Reviewer A is an expert and certified as a specialist in diagnostic radiology and nuclear medicine. Reviewer B was training to be a specialist in diagnostic radiology. Final interpretation as determined by mutual agreement. Images were viewed on a liquid crystal display color monitor, and reviewers could freely change the window level and width.

\section{Assessment}

The first hot spot thought to be a lymph node visualized along a lymphatic channel from the primary tumor site was considered a sentinel lymph node, regardless of injection site. The second hot spot visualized along the same lymph channel was considered a second echelon lymph node (Miura et al. 2010). If the sentinel lymph node and second echelon lymph node existed in the same site, both were regarded as sentinel lymph nodes (Figure 1). Based on these rules, visualized hot spots were assessed to be sentinel lymph nodes by reviewers 
Table 1 Patient characteristics

\begin{tabular}{|c|c|c|c|c|c|c|}
\hline & Patient & Age $(y)$ & Gender & Side & Primary tumor site & Pathology \\
\hline \multirow[t]{21}{*}{ Lower extremities } & 1 & 62 & M & Right & Heel & Malignant Melanoma \\
\hline & 2 & 79 & M & Right & Hallux & Malignant Melanoma \\
\hline & 3 & 57 & $\mathrm{~F}$ & Right & Hallux & Malignant Melanoma \\
\hline & 4 & 54 & $\mathrm{~F}$ & Left & Hallux & Malignant Melanoma \\
\hline & 5 & 78 & $\mathrm{~F}$ & Right & Planta pedis & Malignant Melanoma \\
\hline & 6 & 82 & $\mathrm{~F}$ & Left & Planta pedis & Malignant Melanoma \\
\hline & 7 & 72 & $\mathrm{~F}$ & Right & Planta pedis & Malignant Melanoma \\
\hline & 8 & 66 & $\mathrm{~F}$ & Left & Heel - planta pedis & Malignant Melanoma \\
\hline & 9 & 77 & M & Left & Planta pedis & Malignant Melanoma \\
\hline & 10 & 76 & $\mathrm{~F}$ & Left & Heel & Malignant Melanoma \\
\hline & 11 & 50 & M & Right & Planta pedis & Squamous cell carcinoma \\
\hline & 12 & 51 & $\mathrm{~F}$ & Left & Planta pedis & Malignant Melanoma \\
\hline & 13 & 57 & $\mathrm{~F}$ & Right & Hallux & Malignant Melanoma \\
\hline & 14 & 51 & M & Left & Heel & Malignant Melanoma \\
\hline & 15 & 84 & $\mathrm{~F}$ & Right & Planta pedis & Malignant Melanoma \\
\hline & 16 & 80 & $\mathrm{~F}$ & Right & Planta pedis & Malignant Melanoma \\
\hline & 17 & 74 & $\mathrm{~F}$ & Left & Heel & Malignant Melanoma \\
\hline & 18 & 69 & M & Right & Hallux & Malignant Melanoma \\
\hline & 19 & 84 & M & Left & Heel & Malignant Melanoma \\
\hline & 20 & 74 & $\mathrm{~F}$ & Right & Heel & Malignant Melanoma \\
\hline & 21 & 72 & M & Left & Planta pedis & Malignant Melanoma \\
\hline \multirow[t]{4}{*}{ Upper Extremities } & 22 & 53 & M & Right & Thumb & Malignant Melanoma \\
\hline & 23 & 63 & $\mathrm{~F}$ & Left & Middle finger & Malignant Melanoma \\
\hline & 24 & 73 & M & Right & Dorsum manus & Squamous cell carcinoma \\
\hline & 25 & 76 & $\mathrm{~F}$ & Right & Forefinger & Malignant Melanoma \\
\hline
\end{tabular}

using only the static planar images. On another day, both static planar and dynamic images were assessed. Reviewers scored the diagnostic confidence value of determined sentinel lymph node as follows: 0, cannot be decided; 1 , possible; 2 , probable; and 3, definitive. Differences of diagnostic confidence values with or without dynamic images were confirmed by their average and Wilcoxson's sign-rank sum test. In this statistical analysis, P-value $<0.05$ was considered significant.

\section{Categorization of lymphatic flow}

Patterns of lymphatic drainage from the skin of the lower extremities were categorized into three different pathways: (a) inguinal type, from the primary site to the groin (although there were plural lymphatic pathways in some cases, all paths drained into the groin, indicating that the sentinel lymph node was situated in the groin); (b) popliteal type, from the primary site to the poples; and (c) inguinal and popliteal type, from the primary site to the groin and from the primary site to the poples (Miura et al. 2010). Similarly, patterns of lymphatic drainage from the skin of the upper extremities were also categorized into three different pathways: (d) axillary type, from the primary site to the axilla; (e) cubital type, from the primary site to the cubitus; and (f) the axillary and cubital type, from the primary site to axilla and cubitus (Figure 2).

\section{Results}

\section{Lower extremities}

Reviewer A changed the sentinel lymph node assessment in three of the 21 cases with observation of dynamic images. Reviewer B changed the sentinel lymph node assessment in one of the 21 cases with observation of dynamic images. But there were no cases in which a decision was changed in the same way between reviewer A and B. Diagnostic confidence values with the observation of dynamic images scored by reviewer $\mathrm{A}$ 
a)

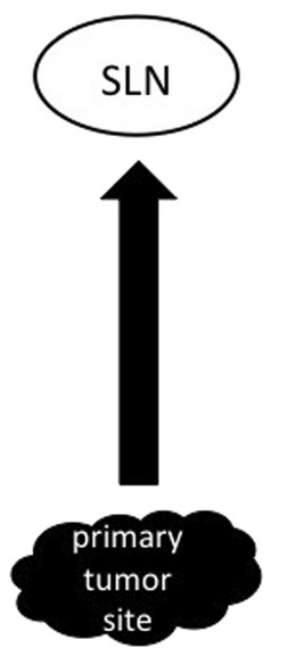

b)

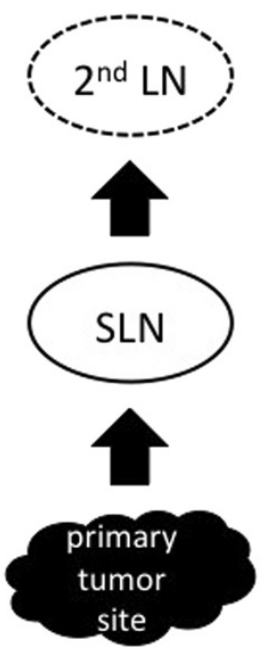

c)

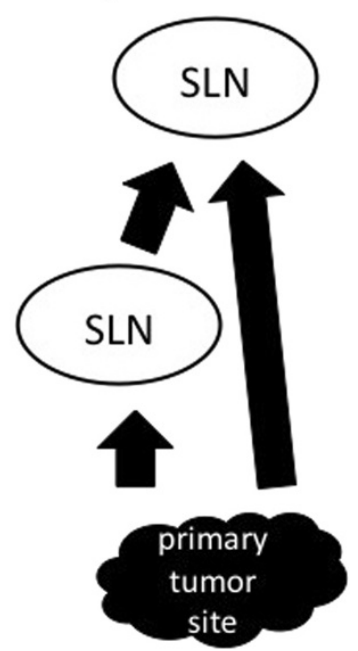

Figure 1 Diagrams of the rules for the sentinel lymph node (SLN) assessment. a) First hot spot thought to be a lymph node (LN) visualized along a lymphatic channel from the primary tumor site was considered a SLN, regardless of injection site. b) Second hot spot visualized along the same lymph channel was considered a second echelon lymph node. c) If the SLN and second echelon LN existed in the same site, both regarded as SLNs.

were increased in five of 21 cases, and decreased in one case. Diagnostic confidence values with the observation of dynamic images scored by reviewer B were increased in four of 21 cases, and there were no decreased cases (Table 2). Average diagnostic confidence values with observation on dynamic images were higher than that without dynamic images except for cases in which popliteal lymph nodes were determined to be sentinel lymph nodes by reviewer A. However, significant differences as calculated by the Wilcoxson's sign-rank sum test were not present (Table 3).

\section{Upper extremities}

Both reviewer A and B changed assessment of the sentinel lymph node in one patient (but not the same case). Diagnostic confidence values with observation of the dynamic images scored by both reviewer A and B were increased in one case (Table 4). Average diagnostic confidence values for cases in which axillary lymph nodes were determined to be the sentinel lymph node were increased with both reviewer A and B, but cases in which cubital lymph nodes were determined to be the sentinel lymph node were same in both reviewers. The number of cases was too small to calculate $P$-values with diagnostic confidence (Table 3 ).

\section{Interpretation by mutual agreement}

In one in 21 cases in the lower extremities, the sentinel lymph node decision was changed with observation of dynamic images (an inguinal and popliteal type). On the other hand, two of four cases in the upper extremities, sentinel lymph node assessment was changed with observation of dynamic images (axillary and cubital type in both cases) (Table 5).

In cases in the lower extremities, lymphatic patterns were categorized as follows: 14 inguinal type (67\%), one popliteal type (5\%), and six inguinal and popliteal type (28\%) (Miura et al. 2010). In cases in the upper extremities, they were categorized as follows: one axillary type (25\%), one cubital type (25\%), and two axillary and cubital types (50\%) (Figure 3).

\section{Differentiation of sentinel lymph node determination with and without dynamic images}

Reviewer A (an expert radiologist) changed the sentinel lymph node assessment with observation of dynamic images in four of 25 cases. Reviewer A assessed the poples, cubitus, and groin (two cases) as sentinel lymph node sites with observation of static images only. They were changed in the following way with observation of static and dynamic images: groin to groin and poples, axilla to axilla and cubitus, and groin to groin and external iliac region. Concerning case No. 17, a lymphatic pathway without popliteal lymph node visualization was observed only on the early phase dynamic images. Similarly, with case No. 24, a lymphatic channel without cubital lymph node visualization was observed only on the 


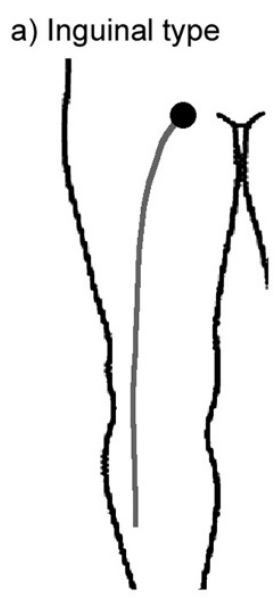

d) Axillary type

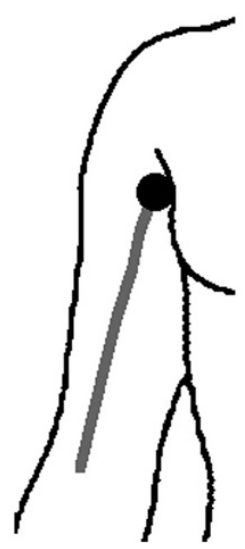

b) Popliteal type

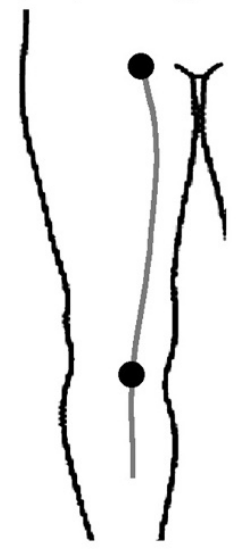

e) Cubital type

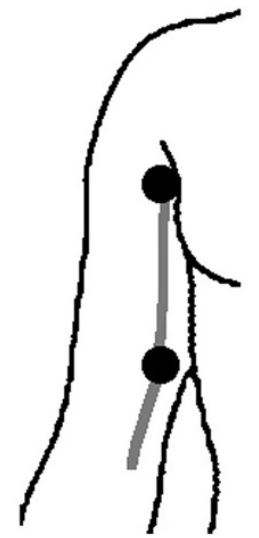

c) Inguinal \& popliteal type

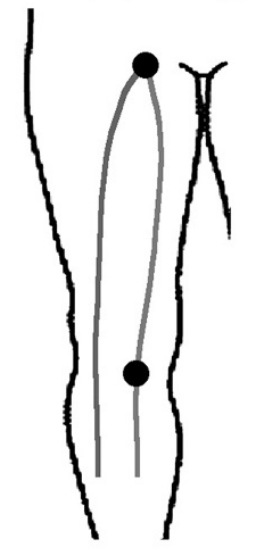

f) Axillary \& cubital type

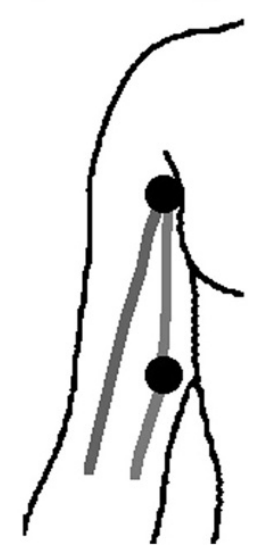

Figure 2 Diagrams of the patterns of the categorized lymphatic drainage of the lower and upper extremities. a) Inguinal type: primary tumor site to groin. b) Popliteal type: primary tumor site to poples. c) Inguinal and popliteal type: primary tumor site to groin and poples. d) Axillary type: primary site to axilla. e) Cubital type: primary site to cubitus. f) Axillary and cubital type: primary site to axilla and cubitus.

early phase dynamic images. Reviewer A noticed these lymphatic channels with observation of dynamic images, and changed the sentinel lymph node assessment (Figure 4).

On the other hand, reviewer B, a radiologist in training, changed sentinel lymph node assessment with observation of dynamic images in two of 25 cases. Reviewer B assessed the groin and poples and assessed the axilla and cubitus as sentinel lymph node sites with observation of static images only, and they were changed to poples only, and cubitus only, respectively. On these images, case No. 1 and No. 24, two lymphatic channels were close to the interval node, and reviewer B might have been uncertain as to if both lymphatic channels passed interval node or not (Figure 5). Interestingly, there were no cases in which an assessment was changed to reflect the same opinion of reviewer $\mathrm{A}$ and $\mathrm{B}$.

\section{Discordance in sentinel lymph node assessment, reviewer} A vs. reviewer $B$

1) Sentinel lymph node classification

Concerning cases No. 1, 16, and 17, reviewer A assessed the sentinel lymph nodes as being on the groin and poples (and external iliac), but reviewer $\mathrm{B}$ assessed the node on the poples only. Concerning cases No. 15 and 19, reviewer A assessed the sentinel lymph nodes as being on the groin and poples (and external iliac), but reviewer B assessed the nodes as being on the groin only. Concerning cases No. 23 and 24, reviewer A assessed the sentinel lymph nodes as being on the axilla and cubitus, but reviewer B assessed the nodes as being the cubitus or brachium.

2) Sentinel lymph node identification 
Table 2 Sentinel lymph node assessment and diagnostic confidence values in cases in the lower extremities

\begin{tabular}{|c|c|c|c|c|c|c|c|c|c|c|c|c|c|c|}
\hline \multirow[t]{4}{*}{ Patient } & \multicolumn{7}{|c|}{ Reviewer A (expert radiologist) } & \multicolumn{7}{|c|}{ Reviewer B (trainee radiologist) } \\
\hline & \multicolumn{6}{|c|}{ Diagnostic confidence value } & \multirow[t]{3}{*}{ Assessment } & \multicolumn{6}{|c|}{ Diagnostic confidence value } & \multirow[t]{3}{*}{ Assessment } \\
\hline & \multicolumn{3}{|c|}{ Only static } & \multicolumn{3}{|c|}{ Static \& dynamic } & & \multicolumn{3}{|c|}{ Only static } & \multicolumn{3}{|c|}{ Static \& dynamic } & \\
\hline & Groin & Poples & External iliac & Groin & Poples & External iliac & & Groin & Poples & External iliac & Groin & Poples & External iliac & \\
\hline 1 & 1 & 2 & & 2 & 3 & & 1 & 1 & 3 & & & 3 & & C \\
\hline 2 & 3 & & & 3 & & & & 3 & & & 3 & & & \\
\hline 3 & 3 & & & 3 & & 3 & C & 3 & & & 3 & & & \\
\hline 4 & 3 & & & 3 & & & & 3 & & & 3 & & & \\
\hline 5 & 3 & & & 3 & & & & 3 & & & 3 & & & \\
\hline 6 & 1 & & & 3 & & & I & 2 & & & 2 & & & \\
\hline 7 & 3 & & & 3 & & 2 & C & 3 & & & 3 & & & \\
\hline 8 & 3 & 3 & & 3 & 3 & & & 1 & 3 & & 3 & 3 & & 1 \\
\hline 9 & 3 & & & 3 & & & & 3 & & & 3 & & & \\
\hline 10 & 3 & & & 3 & & & & 3 & & & 3 & & & \\
\hline 11 & 3 & & & 3 & & & & 3 & & & 3 & & & \\
\hline 12 & 1 & 3 & & 3 & 3 & & 1 & 2 & 2 & & 3 & 3 & & I \\
\hline 13 & 3 & & & 3 & & & & 3 & & & 3 & & & \\
\hline 14 & 3 & 3 & & 3 & 3 & & & 2 & 3 & & 3 & 3 & & I \\
\hline 15 & 3 & 3 & 2 & 3 & 3 & 3 & I & 3 & & & 3 & & & \\
\hline 16 & 1 & 3 & & 3 & 3 & & 1 & & 3 & & & 3 & & \\
\hline 17 & & 3 & & 3 & 3 & & C & & 3 & & & 3 & & \\
\hline 18 & 3 & & & 3 & & & & 3 & & & 3 & & & \\
\hline 19 & 3 & 3 & & 3 & 1 & & D & 3 & & & 3 & & & \\
\hline 20 & 3 & & & 3 & & & & 3 & & & 3 & & & \\
\hline 21 & 3 & & & 3 & & & & 2 & & & 3 & & & I \\
\hline Sentinel node sites & $20 / 21$ & $8 / 21$ & $1 / 21$ & $21 / 21$ & $8 / 21$ & $3 / 21$ & & $19 / 21$ & $6 / 21$ & $0 / 21$ & $18 / 21$ & $6 / 21$ & $0 / 21$ & \\
\hline$\%$ & 95.2 & 38.1 & 4.8 & 100 & 38.1 & 14.3 & & 90.5 & 28.6 & 0 & 85.7 & 28.6 & 0 & \\
\hline
\end{tabular}

C: Assessment of sentinel lymph node was changed with observation of dynamic images.

I: Diagnostic confidence value increased with the observation of dynamic images.

D: Diagnostic confidence value declined with the observation of dynamic images. 
Table 3 Average and P-values* of diagnostic confidence values in cases in the lower \& upper extremities

\begin{tabular}{|c|c|c|c|c|c|c|c|c|c|}
\hline & & \multicolumn{4}{|c|}{$\begin{array}{l}\text { Cases in which inguinal nodes were } \\
\text { determined to be sentinel node }\end{array}$} & \multicolumn{4}{|c|}{$\begin{array}{l}\text { Cases in which popliteal nodes were } \\
\text { determined to be sentinel node }\end{array}$} \\
\hline & & \multicolumn{2}{|c|}{ Reviewer A } & \multicolumn{2}{|c|}{ Reviewer B } & \multicolumn{2}{|c|}{ Reviewer A } & \multicolumn{2}{|c|}{ Reviewer B } \\
\hline & & Only static & $\begin{array}{l}\text { Static \& } \\
\text { dynamic }\end{array}$ & Only static & $\begin{array}{l}\text { Static \& } \\
\text { dynamic }\end{array}$ & Only static & $\begin{array}{l}\text { Static \& } \\
\text { dynamic }\end{array}$ & Only static & $\begin{array}{l}\text { Static \& } \\
\text { dynamic }\end{array}$ \\
\hline \multirow[t]{2}{*}{ Lower extremities } & Average & 2.6 & 2.95 & 2.67 & 2.94 & 2.88 & 2.75 & 2.83 & 3 \\
\hline & $P$-value* & \multicolumn{2}{|c|}{0.059} & \multicolumn{2}{|c|}{0.059} & \multicolumn{2}{|c|}{0.655} & \multicolumn{2}{|c|}{ (Too few to calculate) } \\
\hline Upper extremities & Average & 2.5 & 3 & 2 & 3 & 3 & 3 & 3 & 3 \\
\hline
\end{tabular}

*Wilcoxson's sign-rank sum test.

Concerning cases No. 3 and 7, reviewer A identified the sentinel lymph nodes as being on the groin and external iliac region, but reviewer B identified the nodes as being on the groin only. Concerning cases No. 24 and 25 , reviewer A identified the sentinel lymph nodes as being on the cubitus, but reviewer B identified the nodes as being on the brachium.

\section{Discussion}

In different countries, various radiopharmaceuticals are used for radioguided sentinel LN detection (Glass et al. 1998). In Japan, mainly two radiopharmaceuticals, ${ }^{99 m}$ Tc-labeled tin colloid and ${ }^{99 \mathrm{~m}}$ Tc-labeled phytate, are the primary agents used in sentinel lymphoscintigraphy for malignant skin neoplasms (Higashi et al. 2003). Although ${ }^{99 \mathrm{~m}}$ Tc-phytate is not a colloid, it combines with calcium in vivo and forms a colloid. The particle size of the phytate colloid varies according to the calcium concentration (Higashi et al. 2003), and its ranges from 200 to $1000 \mathrm{~nm}$ (Higashi et al. 2009). Therefore, dynamic scanning using ${ }^{99 m}$ Tc-phytate makes it possible to visualize not only the SLNs but also the second echelon nodes and lymphatic channels (Miura et al. 2010).

A sentinel lymph node is defined as the first lymph node in a lymph node bed to receive lymphatic drainage from a tumor (Even-Sapir et al. 2003). A sentinel lymph node is not just the first node seen on dynamic imaging, because there may be multiple separate lymph channels that have different rates of lymph flow. If these channels drain to different nodes, then all of these nodes are sentinel lymph nodes, regardless of the time taken for the lymph containing the radiocolloid to reach them. A sentinel lymph node is also not necessarily the node closest to the primary site. Lymphatic vessels can bypass many nodes before reaching the sentinel lymph node (Uren et al. 2003). Therefore, sentinel lymph nodes are not always regional lymph nodes, and sentinel lymph nodes and second echelon lymph nodes may exist on the same site. Conversely, if it is possible to confirm interval node is sentinel lymph node and regional lymph node is not sentinel lymph node, interval node biopsy alone may be sufficient. However, assessment of sentinel lymph nodes of the upper and lower extremities was often different between the reviewers.

a) Differences in sentinel lymph node assessment using dynamic images: difficulties in observation of dynamic images

Differences in sentinel lymph node assessment using dynamic images were decided by both reviewer A and B to exist between "inguinal \& popliteal type" and "axillary \& cubital type" nodes. In these cases, lymphatic channels existed that did not pass through the interval lymph node. In addition, lymphatic channels observed solely on early phase dynamic images. Therefore, if lymphatic channels that did not pass through the interval lymph node were visualized, even in a short period, lymph nodes into which both lymphatic channels flowed into were regarded as sentinel lymph nodes. Reviewer A, an expert diagnostic radiologist noted lymphatic channels visualized only by dynamic images, and changed the sentinel lymph node assessment. However, reviewer B, a trainee radiologist was not aware of the lymphatic channels visualized only by dynamic images, and did not change the sentinel lymph node assessment. These lymphatic channels were only observed during a short period (Miura et al. 2010; Glass et al. 1998), and were not clearly visualized because of faint radioactivity. In some cases, it is difficult to determine if lymphatic channels flow into lymph nodes or just overlap lymph nodes. However, clinical recurrence might also occur in interval nodes that are sentinel lymph nodes if they are not subjected to biopsy during initial surgical treatment, and interval nodes not be overlooked if the sentinel lymph node biopsy procedure is to be as accurate as possible in all patients (Uren et al. 2000). Therefore, careful observation with repetition and adjustment of window level and width is enormously needed.

b) Diagnostic confidence values of sentinel lymph node assessment of the lower extremities 
Table 4 Sentinel lymph node assessment and diagnostic confidence values in cases in the upper extremities

\begin{tabular}{|c|c|c|c|c|c|c|c|c|c|c|c|c|c|c|}
\hline \multirow[t]{4}{*}{ Patient } & \multicolumn{7}{|c|}{ Reviewer A (expert radiologist) } & \multicolumn{7}{|c|}{ Reviewer B (trainee radiologist) } \\
\hline & \multicolumn{6}{|c|}{ Diagnostic confidence value } & \multirow[t]{3}{*}{ Assessment } & \multicolumn{6}{|c|}{ Diagnostic confidence value } & \multirow[t]{3}{*}{ Assessmen } \\
\hline & \multicolumn{3}{|c|}{ Only static } & \multicolumn{3}{|c|}{ Static \& dynamic } & & \multicolumn{3}{|c|}{ Only static } & \multicolumn{3}{|c|}{ Static \& dynamic } & \\
\hline & Axilla & Cubitus & Brachium & Axilla & Cubitus & Brachium & & Axilla & Cubitus & Brachium & Axilla & Cubitus & Brachium & \\
\hline 22 & 3 & & & 3 & & & & 3 & & & 3 & & & \\
\hline 23 & 2 & 3 & & 3 & 3 & & 1 & 1 & 3 & & & 3 & & C \\
\hline 24 & & 3 & & 3 & 3 & & C & & & 1 & & & 3 & 1 \\
\hline 25 & & 3 & & & 3 & & & & & 3 & & & 3 & \\
\hline Number of sentinel sites & $2 / 4$ & $3 / 4$ & $0 / 4$ & $3 / 4$ & $3 / 4$ & $0 / 4$ & & $2 / 4$ & $1 / 4$ & $2 / 4$ & $1 / 4$ & $1 / 4$ & $2 / 4$ & \\
\hline$\%$ & 50 & 75 & 0 & 75 & 75 & 0 & & 50 & 25 & 50 & 25 & 25 & 50 & \\
\hline
\end{tabular}

C: Assessment of sentinel lymph node was changed with observation of dynamic images.

I: Diagnostic confidence value increased with the observation of dynamic images. 
Table 5 Assessment of SLN in the upper and lower extremities by mutual agreement

\begin{tabular}{|c|c|c|c|c|c|c|c|c|c|c|c|c|c|}
\hline \multicolumn{7}{|c|}{ A Lower extremities } & \multicolumn{7}{|c|}{ B Upper extremities } \\
\hline \multirow[t]{3}{*}{ Patient } & \multicolumn{4}{|c|}{ Assessment } & \multirow[t]{3}{*}{ Change } & \multirow{3}{*}{$\begin{array}{c}\text { Category } \\
\text { of lymphatic } \\
\text { pattern* }\end{array}$} & \multirow[t]{3}{*}{ Patient } & \multicolumn{4}{|c|}{ Assessment } & \multirow[t]{3}{*}{ Change } & \multirow{3}{*}{$\begin{array}{c}\text { Category } \\
\text { of lymphatic } \\
\text { pattern** }\end{array}$} \\
\hline & \multicolumn{2}{|c|}{ Only static } & \multicolumn{2}{|c|}{$\begin{array}{c}\text { Static \& } \\
\text { dynamic }\end{array}$} & & & & \multicolumn{2}{|c|}{ Only static } & \multicolumn{2}{|c|}{$\begin{array}{l}\text { Static \& } \\
\text { dynamic }\end{array}$} & & \\
\hline & Groin & Poples & Groin & Poples & & & & Axilla & Cubitus & Axilla & Cubitus & & \\
\hline 1 & 2nd & SLN & 2nd & SLN & No & $P$ & 22 & SLN & & SLN & & No & A \\
\hline 2 & SLN & & SLN & & No & I & 23 & 2nd & SLN & SLN & SLN & Yes & $A C$ \\
\hline 3 & SLN & & SLN & & No & 1 & 24 & 2nd & SLN & SLN & SLN & Yes & $A C$ \\
\hline 4 & SLN & & SLN & & No & 1 & 25 & 2nd & SLN & 2nd & SLN & No & $C$ \\
\hline 5 & SLN & & SLN & & No & I & $\begin{array}{l}\text { Number of } \\
\text { SLN sites }\end{array}$ & $2 / 4$ & $3 / 4$ & $2 / 4$ & $3 / 4$ & & \\
\hline 6 & SLN & & SLN & & No & 1 & $\%$ & 50 & 75 & 50 & 75 & & \\
\hline 7 & SLN & & SLN & & No & I & & & & & & & \\
\hline 8 & SLN & SLN & SLN & SLN & No & $\mathbb{I P}$ & & & & & & & \\
\hline 9 & SLN & & SLN & & No & 1 & & & & & & & \\
\hline 10 & SLN & & SLN & & No & 1 & & & & & & & \\
\hline 11 & SLN & & SLN & & No & 1 & & & & & & & \\
\hline 12 & SLN & SLN & SLN & SLN & Yes & $\mathbb{I P}$ & & & & & & & \\
\hline 13 & SLN & & SLN & & No & 1 & & & & & & & \\
\hline 14 & SLN & SLN & SLN & SLN & No & $\mathbb{I P}$ & & & & & & & \\
\hline 15 & SLN & & SLN & & No & 1 & & & & & & & \\
\hline 16 & SLN & SLN & SLN & SLN & No & $\mathbb{I P}$ & & & & & & & \\
\hline 17 & 2nd & SLN & SLN & SLN & Yes & $\mathbb{I P}$ & & & & & & & \\
\hline 18 & SLN & & SLN & & No & 1 & & & & & & & \\
\hline 19 & SLN & SLN & SLN & SLN & No & $\mathbb{I P}$ & & & & & & & \\
\hline 20 & SLN & & SLN & & No & 1 & & & & & & & \\
\hline 21 & SLN & & SLN & & No & 1 & & & & & & & \\
\hline $\begin{array}{l}\text { Number of } \\
\text { SLN sites }\end{array}$ & $17 / 21$ & $7 / 21$ & $20 / 21$ & $7 / 21$ & & & & & & & & & \\
\hline$\%$ & 81 & 33.3 & 95.2 & 33.3 & & & & & & & & & \\
\hline
\end{tabular}

SLN: sentinel lymph node.

2nd: second echelon lymph node.

*I: inguinal type.

*P: popliteal type.

*IP: inguinal and popliteal type.

${ }^{* *}$ A: axillary type.

${ }^{* *} \mathrm{C}$ : cubital type.

${ }^{* *}$ AC: axillary and cubital type.

In most cases, average diagnostic confidence values with observation of dynamic images for both reviewer $\mathrm{A}$ and $B$ were higher than that without observation of dynamic images. However, significant differences were not present. In many cases, information about lymphatic flow was added with observation of dynamic images (Miura et al. 2010). Dynamic images were also useful when a hot spot could not be determined to be a lymph node, or a so-called "lymphatic lake", that is, focal dilatation of lymphatic collecting vessel (Uren et al. 2003; Miura et al. 2010). However, too few upper extremities were analyzed in order to calculate diagnostic confidence value in the present study.

c) Categorization of visualized lymphatic patterns

In cases of the lower extremities, the "popliteal type" was found in one of 21 cases, only $5 \%$. This fact may indicate that lymphatic patterns are limited in cases in which the popliteal lymph node is the sentinel lymph node, and all inguinal lymph nodes are second echelon lymph nodes (Miura et al. 2010; Kutsuna 1968). 


\section{a) Lower extremities}

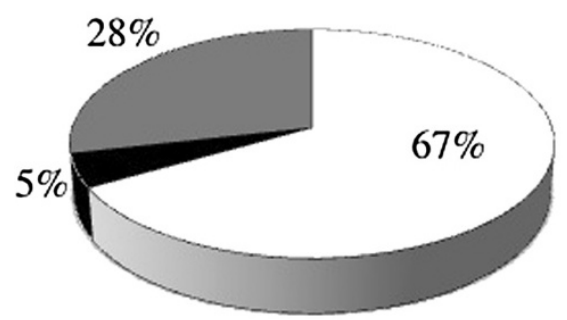

$\square$ Ingiunal type: 14 cases

- Popliteal type: 1 case

${ }^{\square}$ Popliteal \& inguinal type: 6 cases

\section{b) Upper extremities}

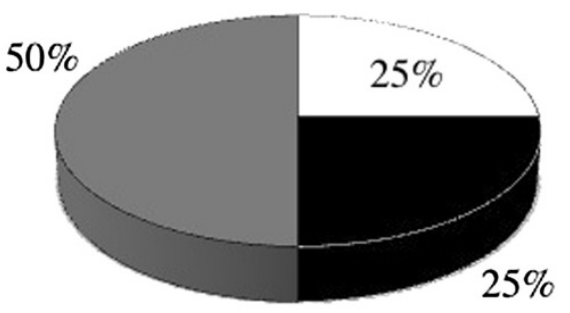

$\square$ Axillary type: 1 case

- Cubital type: 1 case

$\square$ Axillary \& cubital type: 2 cases

Figure 3 Patterns of lymphatic drainage in cases of malignant skin neoplasms in the lower and upper extremities interpreted by mutual agreement. a) In cases of the lower extremities: 14 inguinal type (67\%), one popliteal type (5\%), and 6 inguinal and popliteal type (28\%). b) In cases of the upper extremities: one axillary type (25\%), one cubital type (25\%), and 2 axillary and cubital type (50\%).

In cases of the upper extremities, the "cubital type" was one of 4 cases, or $25 \%$. There may possibly be many lymphatic patterns in which the cubital lymph node is the sentinel lymph node, and all axillary lymph nodes are second echelon lymph nodes. However, there were not enough cases of the upper extremities in the present study to conclude this.

d) Trustworthiness of dynamic images
There are many problems with dynamic images of sentinel lymphoscintigraphy. Because of the faint radioactivity of lymphatic vessels, visualization is often obscure: therefore, whether or not all lymphatic channels are visualized is questionable. Furthermore, lymphatic drainage often flows outside the image acquisition time. Overlapping lymphatic vessels often prevent comprehension of the correct lymphatic direction (Matter et al. 2007). Differentiation between a lymph node and a "lymphatic lake" (Uren a)

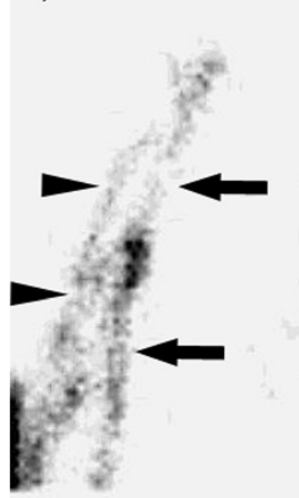

b)

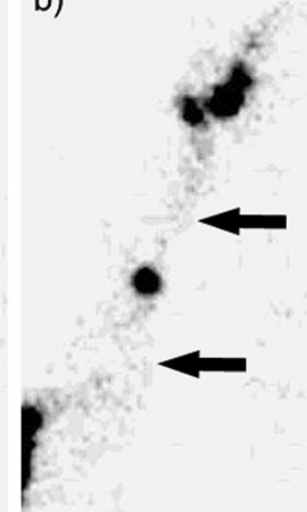

c)

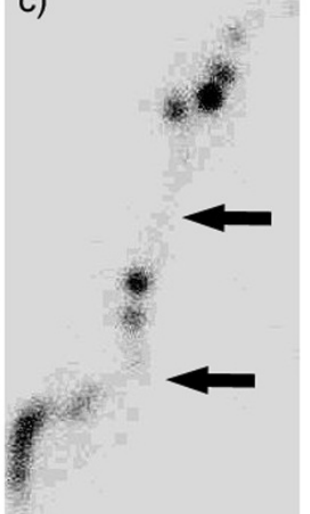

d)

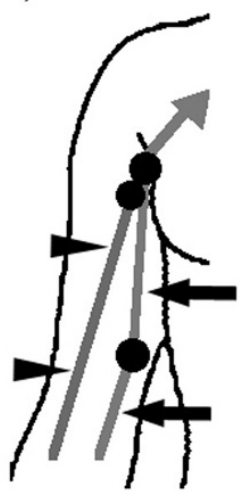

Figure 4 A case of malignant melanoma of the right dorsum manus. (a) Early-phase dynamic image (within 2 min of radiotracer injection) showed that lymphatic channels both passed through the cubitus (black arrow), and did not pass through the cubitus (black arrowheads). Therefore, both cubital and axillary LNs were diagnosed as SLNs. (b) Late-phase dynamic image (about 10 min after radiotracer injection) showed the lymphatic channel passing only through the cubitus (black arrow). (c) Static image about 20 min after tracer injection also showed the only lymphatic channel passing through the cubitus (black arrow). (d) Diagram of early-phase dynamic image. Without a dynamic scan, and without awareness of the lymphatic drainage only visualized on early-phase dynamic images, the axillary LN would have been incorrectly diagnosed as the second echelon LN. 
a)

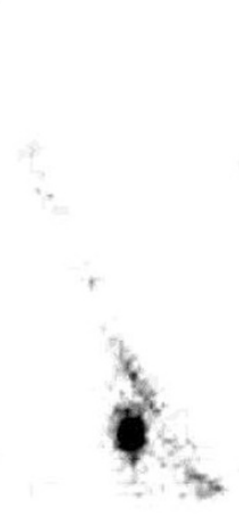

b)

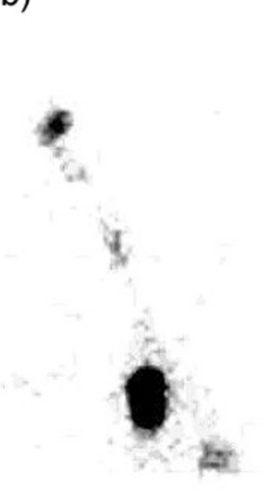

c)

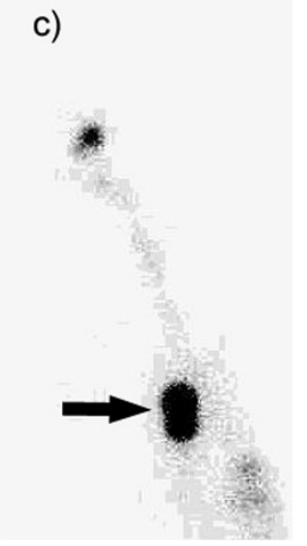

d)

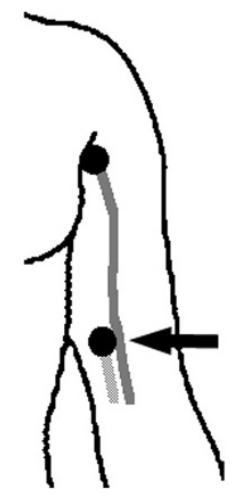

Figure 5 A case of malignant melanoma of the left middle finger. (a) Early-phase dynamic image showed a hot spot thought to be LN on the cubitus (black arrow). (b) Late-phase dynamic image showed the lymphatic channel to the axilla and a hot spot thought to be LN on the axillary region. (c) Static image about 20 min after tracer injection showed the hot spots on both cubitus (black arrow) and axilla, and lymphatic channels to the cubitus and axilla. (d) Diagram of late-phase dynamic image and static image. It is difficult to assess both lymphatic channels pass through the axillary LN (black arrow) or not because of blurred images.

et al. 2003; Matter et al. 2007) is sometimes difficult. Lymphatic lakes should not be mistaken for interval nodes, which retain tracer and are hot on delayed scans (Uren et al. 2003). Therefore, in the present circumstances, all biopsies of the hot spots determined to be lymph nodes should not be excluded in order to avoid the risk of undertreatment (Matter et al. 2007). However, excessive biopsies should be avoided as much as possible from the viewpoint of the sentinel lymph node concept (Pijpers et al. 1997). Many problems surrounding these issues remain to be resolved.

\section{Conclusions}

The present study examined whether or not the use of dynamic images alters the outcome of sentinel lymph node assessment in patients with malignant skin neoplasms of the upper and lower extremities. Some lymphatic channels were observed using early phase dynamic images only, and sentinel lymph node assessment was altered in some cases. Determination of whether a lymph node is a sentinel lymph node depends on visualization of the lymphatic network. In the cases of the lower extremities, the average diagnostic confidence value of sentinel lymph node assessment is usually higher with observation of dynamic images. In many cases, information about lymphatic flow was added with observation of dynamic images and it was helpful for sentinel lymph node assessment. It is necessary to use dynamic images alongside skillful observation in assessment of sentinel lymph nodes.

\section{Competing interests}

The authors declare that they have no competing interests.

\section{Authors' contributions}

$\mathrm{HM}$ has planned the study design, collected the data of the patients, made the analysis of the results, and written the manuscript. SO and HS were image reviewers in this study, and supported the collection of the data. TK and SD performed patient management and directed the manuscript from viewpoints of specialists of dermatology. $Y T$ directed the design of the study and the manuscript. KS, FT, SK, KH, HF, AK, MA, YH, HK, and MS have supported the collection of the data of the patients and analysis of the results. All authors read and approved the final manuscript.

\section{Acknowledgement}

This research was awarded a Cypos Gold Medal at the 70th Annual Meeting of the Japan Radiological society.

\section{Author details}

${ }^{1}$ Department of Radiology, Hirosaki University School of Medicine, 5 Zaifu-cho, Hirosaki, Aomori 036-8562, Japan. ²Department of Dermatology, Hirosaki University School of Medicine, Hirosaki, Japan.

Received: 1 August 2014 Accepted: 18 September 2014 Published: 22 October 2014

\section{References}

Alazraki N, Glass EC, Castronovo F, Valdès Olmos RA, Podoloff D (2002) Procedure guideline for lymphoscintigraphy and the use of intraoperative gamma probe for sentinel lymph node localization in melanoma of intermediate thickness 1.0. J Nucl Med 43:1414-1418

Belhocine T, Piérard GE, Gielen JL, Daenen F, De Barsy C, Arrese JE, Lahaye T, Rigo P (2002) Gamma-probe-directed lymphatic mapping and sentinel lymphadenectomy in primary cutaneous melanoma. Dermatology 204:355-361

Even-Sapir E, Lerman H, Lievshitz G, Khafir A, Fliss D, Schwartz A, Gur E, Schneebaum S (2003) Lymphoscintigraphy for sentinel node mapping using a hybrid SPECT/CT system. J Nucl Med 44:1413-1420

Gennari R, Bartolomei M, Testori A, Zurrida S, Stoldt HS, Audisio RA, Geraghty JG, Paganelli G, Veronesi U (2000) Sentinel node localization in primary 
melanoma: Preoperative dynamic lymphoscintigraphy, intraoperative gamma probe, and vital dye guidance. Surgery 127:19-25

Glass EC, Essner R, Morton DL (1998) Kinetics of three lymphoscintigraphic agents in patients with cutaneous melanoma. J Nucl Med 39:1185-1190

Higashi H, Natsugoe S, Uenosono Y, Ehi K, Arigami T, Nakabeppu Y, Nakajo M, Aikou T (2003) Particle size of tin and phytate colloid in sentinel node identification. J Surg Res 121:1-4

Higashi U, Takata M, Saida T (2009) Sentinel lymph node biopsy in Japan. Int J Clin Oncol 14:490-496

Jacobs IA, Chevinsky AH, Swayne LC, Magidson JG, Britto EJ, Smith TJ (2001) Gamma probe-directed lymphatic mapping and sentinel lymphadenectomy in primary melanoma: reliability of the procedure and analysis of failures after long-term follow-up. J Surg Oncol 77:157-164

Kutsuna M (1968) Anatomie des Lymphsystems der Japaner. Kanehara Shuppan Co, Tokyo

Mariani G, Gipponi M, Moresco L, Villa G, Bartolomei M, Mazzarol G, Bagnara MC, Romanini A, Cafiero F, Paganelli G, Strauss W (2002) Radioguided sentinel lymph node biopsy in malignant cutaneous melanoma. J Nucl Med 43:811-827

Mariani G, Ebra P, Manca G, Villa G, Gipponi M, Boni G, Buffoni F, Suriano S, Castagnola F, Bartolomei M, Strauss HW (2004) Radioguided sentinel lymph node biopsy in patients with malignant cutaneous melanoma: the nuclear medicine contribution. J Surg Oncol 85:141-151

Matter M, Lalonde MN, Allaoua M, Boubaker A, Li'enard D, Gugerli O, Cerottini JP, Bouzourene $H$, Delaloye AB, Lejeune $F$ (2007) The role of interval nodes in sentinel lymph node mapping and dissection for melanoma patients. J Nucl Med 48:1607-1613

Maza S, Valencia R, Geworski L, Sandrock D, Zander A, Audring H, Dräger E, Winter H, Sterry W, Munz DL (2003) Influence of fast lymphatic drainage on metastatic spread in cutaneous malignant melanoma: a prospective feasibility study. Eur J Nucl Med Mol Imaging 30:538-544

Miura H, Ono S, Nagahata M, Shibutani K, Kakehata S, Morimoto K, Morimoto K, Seino H, Kakuta A, Aoki M, Hayayama Y, Kawaguchi H, Takai Y, Abe Y, Kaneko T, Sawamura D, Hanada K (2010) Lymphoscintigraphy for sentinel lymph node mapping in Japanese patients with malignant skin neoplasms of the lower extremities: comparison with previously investigated Japanese lymphatic anatomy. Ann Nucl Med 24:601-608

Murray DR, Carlson GW, Greenlee R, Alazraki N, Fry-Spray C, Hestley A, Poole R, Blais M, Timbert DS, Vansant J (2000) Surgical management of malignant melanoma using dynamic lymphoscintigraphy and gamma probe-guided sentinel lymph node biopsy: the Emory experience. Am Surg 66:763-767

Pijpers R, Collet GJ, Meijer S, Hoekstra OS (1995) The impact of dynamic lymphoscintigraphy and gamma probe guidance on sentinel node biopsy in melanoma. Eur J Nucl Med 22:1238-1241

Pijpers R, Borgstein PJ, Meijer S, Hoekstra OS, van Hattum LH, J. Teule GJ (1997) Sentinel node biopsy in melanoma patients: dynamic lymphoscintigraphy followed by intraoperative gamma probe and vital dye guidance. World J Surg 21:788-793

Rossi CR, De Salvo GL, Trifirò G, Mocellin S, Landi G, Macripò G, Carcoforo P, Ricotti G, Giudice G, Picciotto F, Donner D, Di Filippo F, Montesco MF, Casara D, Schiavon M, Foletto M, Baldini F, Testori A (2006) The impact of lymphoscintigraphy technique on the outcome of sentinel node biopsy in 1,313 patients with cutaneous melanoma: an Italian Multicentric study (SOLISM-IMI). J Nucl Med 47:234-241

Taylor A, Jr, Murray D, Herda S, Vansant J, Alazraki N (1996) Dynamic lymphoscintigraphy to identify the sentinel and satellite nodes. Clin Nucl Med 21:755-758

Tiffet O, Perrot JL, Gentil-Perret A, Prevot N, Dubois F, Alamartine E, Cambazard F (2004) Sentinel lymph node detection in primary melanoma with preoperative dynamic lymphoscintigraphy and intraoperative $\gamma$ probe guidance. Br J Surg 91:886-892

Toubert ME, Just PA, Baillet G, Kerob D, Hindiè E, Verola O, Revol M, Servant JM, Basset-Seguin N, Lebbé C, Banti E, Rubello D, Moretti JL (2008) Slow dynamic lymphoscintigraphy is not a reliable predictor of sentinel-node negativity in cutaneous melanoma. Cancer Biother Radiopharm 23:443-450

Uren RF, Howman-Giles R, Thompson JF, Quinn MJ, O'Brien C, Shaw HM, Bosch CM, McCarthy WH (1996) Lymphatic drainage to triangular intermuscular space lymph nodes in melanoma on the back. J Nucl Med 37:964-966

Uren RF, Howman-Giles R, Thompson JF, McCarthy WH, Quinn MJ, Roberts JM, Shaw HM (2000) Interval nodes - the forgotten sentinel nodes in patients with melanoma. Arch Surg 135:1168-1172
Uren RF, Howman-Giles R, Thompson UF (2003) Patterns of lymphatic drainage from the skin in patients with melanoma. J Nucl Med 44:570-582

Vidal-Sicart S, Pons F, Puig S, Ortega M, Vilalta A, Martin F, Rull R, Palou JM, Castel $T$ (2003) Identification of the sentinel lymph node in patients with malignant melanoma: what are the reasons for mistakes? Eur J Nucl Med Mol Imaging 30:362-366

Vidal-Sicart S, Pons F, Fuertes S, Vilalta A, Rull R, Puig S, Palou JM, Ortega M, Castel T (2004) Is the identification of in-transit sentinel lymph nodes in malignant melanoma patients really necessary? Eur J Nucl Med Mol Imaging 31:945-949

\section{doi:10.1186/2193-1801-3-625}

Cite this article as: Miura et al:: Contribution of dynamic sentinel lymphoscintigraphy images to the diagnosis of patients with malignant skin neoplasms in the upper and lower extremities. SpringerPlus 2014 3:625.

\section{Submit your manuscript to a SpringerOpen ${ }^{\circ}$ journal and benefit from:}

- Convenient online submission

Rigorous peer review

- Immediate publication on acceptance

- Open access: articles freely available online

- High visibility within the field

- Retaining the copyright to your article

Submit your next manuscript at $\gg$ springeropen.com 